Citation: Salas de Armas I. et al. (2019) "Implantation of Left Ventricular Assist Device in the setting of heavily calcified left ventricular apex using an apex preserving technique."

The VAD Journal, 5. doi: https://doi.org/10.13023/vad.2019 .02

Editor-in-Chief: Maya Guglin, University of Kentucky

Received: October 22, 2018

Accepted: January 19, 2019

Published: January 20, 2019

() 2019 The Author(s). This is an open access article published under the terms of the Creative Commons Attribution-

NonCommercial 4.0 International License

(https://creativecommons.org/lice nses/by-nc/4.0/), which permits unrestricted non-commercial use, distribution, and reproduction in any medium, provided that the original author(s) and the publication source are credited.

Funding: Not applicable

Competing interests: Not applicable
Peer-Reviewed Case Report

\section{Implantation of Left Ventricular Assist Device in the setting of heavily calcified left ventricular apex using an apex preserving technique}

\author{
Ismael A Salas de Armas, Mehmet Akay, Jayeshkumar A Patel, \\ Chandni J Patel, Manish K Patel, Bindu Akkanti, Biswajit Kar, and Igor \\ Gregoric*
}

*Corresponding author: Igor.D.Greqoric@uth.tmc.edu

The University of Texas Health Science Center-Houston, Houston, TX, USA 
fashion (Cerclage). The HeartMate II LVAD inflow cannula was secured into the sawing ring, and the rest of the procedure was conducted in the standardized fashion. The patient was discharged into a rehabilitation center eight weeks after LVAD implantation. Thus, if the calcific area is maintained by coring just the inflow site, the spherical shape of the ventricle is maintained to all for better positioning of the inlet cannula. The second suture line enhances hemostasis around the inflow insertion site and stays away from the calcium, which sits in a deeper layer. This procedure, the apex preserving cerclage technique (APCT), does not increase surgical time and reinforces the tissue around the inlet site.

Keywords: VA-ECMO, LVAD, calcification, mechanical circulatory support

\section{Abbreviations:}

CF-LVAD: Continuous Flow Left Ventricular Assist Device

LV: Left Ventricular/Left Ventricle

APCT: Apex Preserving Cerclage Technique

EKG: Echocardiogram

LHC: Left Heart Catheterization

CTO: Chronic Total Occlusion

RCA: Right Coronary Artery

LAD: Left Anterior Descending

VT: Ventricular Tachycardia

IABP: Intra-aortic Balloon Pump

VA-ECMO: Venous-arterial Extracorporeal Membrane Oxygenation

CT: Computed tomography

TEE: Transespophageal Echocardiogram

ICU: Intensive Care Unit

ERCP: Endoscopic Cholangio-Pancreatography

AICD: Automatic Implantable Cardioverter-Defibrillator

\section{Introduction}

Intracorporeal Continuous-Flow Left Ventricular Assist Devices (CF-LVAD) are one of the standard modalities for the treatment of end-stage heart disease either as a bridge to transplantation or as destination therapy (1). The presence of an adequate ventricular apex and ascending aorta for the placement of the inflow cannula and outflow graft, respectively, is paramount. Furthermore, the position of the inflow cannula in the left ventricle (LV) is one of the most important determinants for adequate LVAD function $(2,3)$. When an intracoropreal CF-LVAD is implanted, the only two options for the insertion of the inflow cannula are the true apical or diaphragmatic positions. If the ventricular apex is calcified, this complicates the advanced heart failure surgery.

There have been few papers written in the literature on how to accommodate a heavily calcified ventricular apex. Those papers have suggested mainly excisional 
approaches like LV apical resection and LV reconstruction with a Dacron patch (4); enucleation of large, calcified ventricular aneurysms (5); or extensive LV excision, including the mitral apparatus, to establish a single left cavity (6). Here, a non-excisional approach is described in which the sutures for the inlet cannula are placed around the calcific apex (Apex Preserving) away from the core site, parachuting the inflow sewing ring into an intramyocardial position (Telescope) and assuring hemostasis by placing a felt strip on the epicardial tissue in a purse string fashion (Cerclage). We have called this procedure the apex preserving cerclage technique (APCT).

\section{Case Report and Operative Technique}

A 44-year-old, Asian, male with no significant past medical history, originally presented to an outside hospital with chest pain. Initial assessments revealed elevated troponins and EKG changes. The patient underwent a left heart catheterization (LHC) that showed chronic total occlusion (CTO) of the right coronary artery (RCA) and left anterior descending (LAD) artery dissection. During the procedure, the patient had episodes of ventricular tachycardia (VT) arrest. Initially, an intra-aortic balloon pump (IABP) was placed; it was then upgraded to a femoral venous-arterial percutaneous peripheral extra corporeal membrane oxygenation (VA-ECMO) for adequate hemodynamic support. The patient was transferred to our service for advanced heart failure management and was approved for the implantation of an intracorporeal LVAD as a destination therapy by the institutional medical review board.

Pre-operative assessments included a chest x-ray and computed tomography (CT), which showed a heavily calcified ventricular apex (Fig. 1).

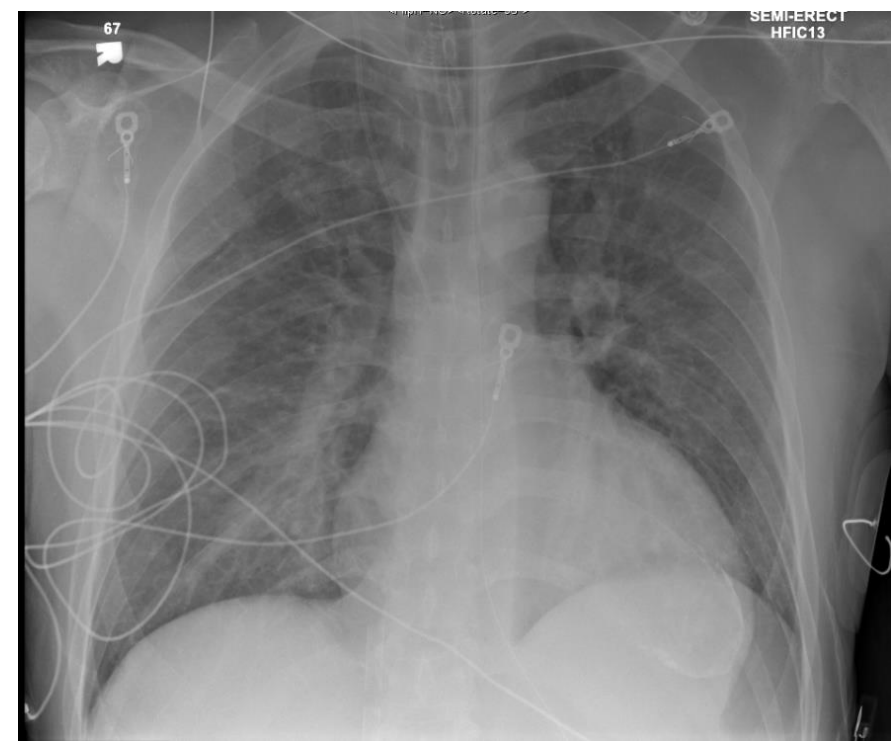

Figure 1. Chest X-Ray, one view. Heavily calcified left ventricular apex.

The patient was taken to the operating room where a standard median sternotomy was followed by the creation of a pre-peritoneal LVAD pocket. Next, a skin-hole 
punch was applied to the right upper quadrant. At this site, a 28 Fr chest tube was tunneled behind the abdominal fascia, through the pre-peritoneal pocket, and exteriorized for the planned path of the LVAD driveline. Systemic heparin was given; peripheral VA-ECMO was switched to central cardiopulmonary bypass (CPB) through aortic and right atrial standard cannulation. The intraoperative transesophageal echo (TEE) showed an intraventricular thrombus. The LV apex was identified and elevated. A HeartMate II coring knife (Abbott, previously, St. Jude-Thoratec) was used initially. There was extensive calcification; a ventriculotomy was completed with heavy scissors (Apex Preserving). The ventricular cavity was inspected and a large number of thrombi were found. We meticulously removed all of the clots. Then, a series of Ethibond (Ethicon, Inc.) interrupted sutures were placed circumferentially around the LV apical cannulation site. Most importantly, stiches were placed around and behind the apical calcified site in a circumferential fashion through a piece of PTFE felt, with minimal gaps between individual suture set and in relation to the LV entry site. Each pair of sutures was placed behind the calcific apex and, subsequently, through the sewing ring of the HeartMate II (Abbott, previously St. Jude-Thoratec) inflow cannula. The sewing ring was then parachuted into position (Fig. 2), such that the sewing ring was intramyocardial to almost an intracavitary location (Telescope). Then, the sutures were sequentially hand tied. Next, the HeartMate II CF-LVAD pump was placed such that the metal inflow arm was engaged into the sewing ring/inflow cannula. Once it was positioned satisfactorily, sutures around the sewing ring were completed and two zip ties were placed (Fig 3). Next, a second circumferential felt strip was sutured to the epicardium adjacent to the initial felt strip using 3-0 prolene sutures in a purse string fashion (Cerclage) (Fig 4).

The externalization of the driveline was guided by the tunneled device, and attention was paid to the performance of the outflow graft. The outflow graft deairing process and initiation of LVAD, as well as weaning from CPB, was conducted in the usual fashion. The ECMO cannulas were removed and the femoral vessels were repaired. Protamine was given, and the chest was left temporarily closed due to coagulopathy. The patient was kept intubated, received minimal inotropic support, and was transferred to the ICU for post-LVAD management. Twenty-four hours later, the patient was brought back to the operating room for mediastinal washout and definitive closure.

Intraoperative TEE and post-operative chest $x$-rays showed adequate positioning of the inflow cannula. The LVAD inlet cannula insertion through the left apical calcification is shown in a 3D CT chest reconstruction (Fig 5).

The post-operative course was remarkable for acute pancreatitis requiring endoscopic retrograde cholangio-pancreatography (ERCP) and intrapancreatic duct stent placement; episodes of ventricular tachycardia (VT) requiring ablation and placement of dual chamber automated internal cardiac defibrillator (AICD); and pneumonia requiring intravenous antibiotics. The patient was finally discharged to a rehabilitation center eight weeks after LVAD implantation. 


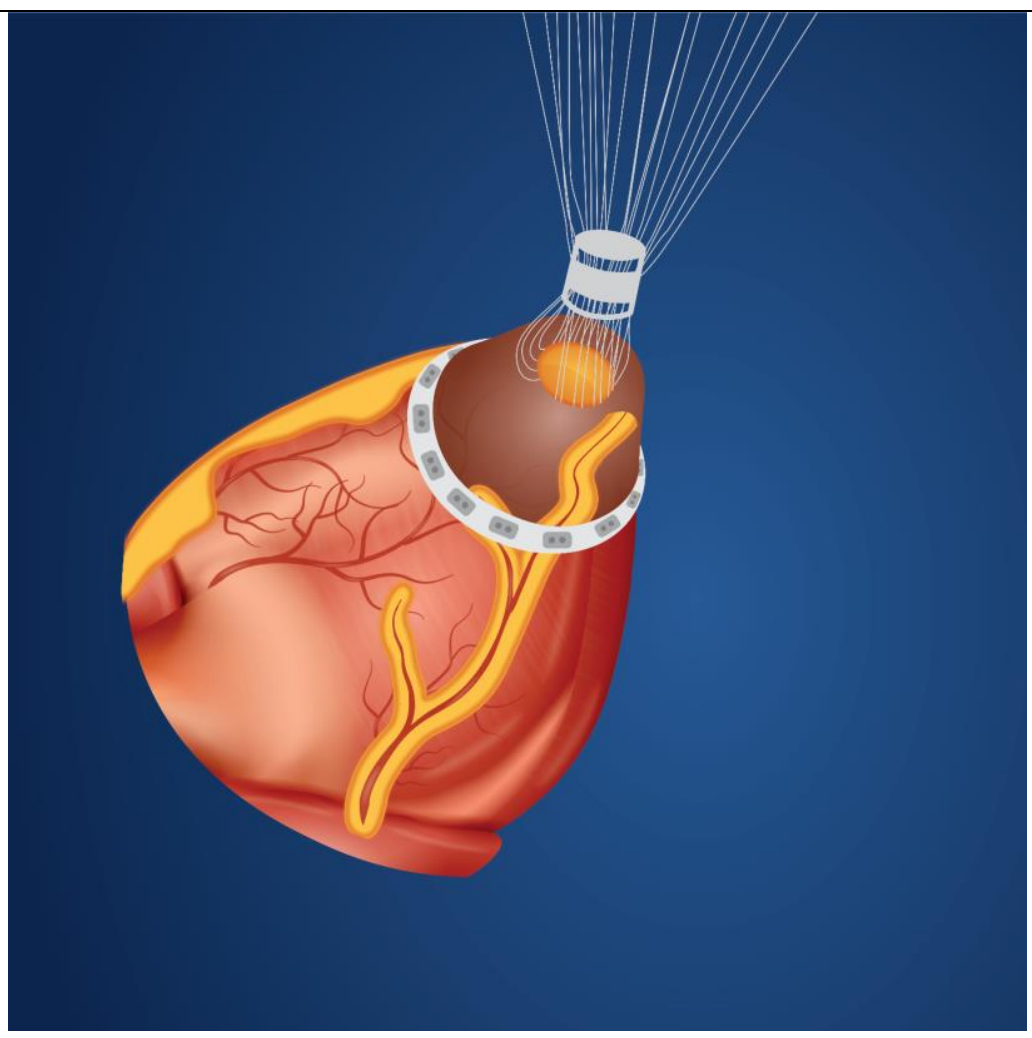

Figure 2. Sewing ring sutures behind the calcific apex parachuting the sewing ring into intracavitary position.

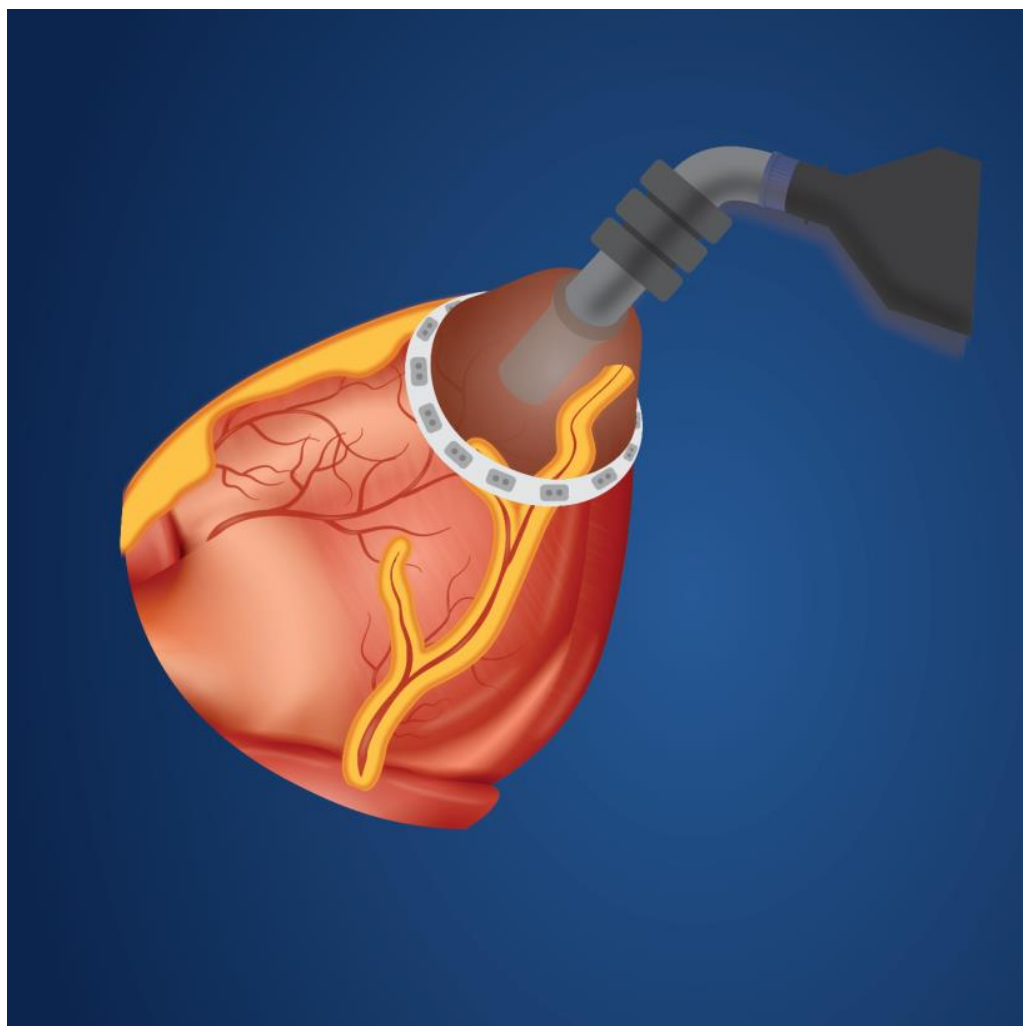

Figure 3. The inflow cannula is connected to the sewing ring. 


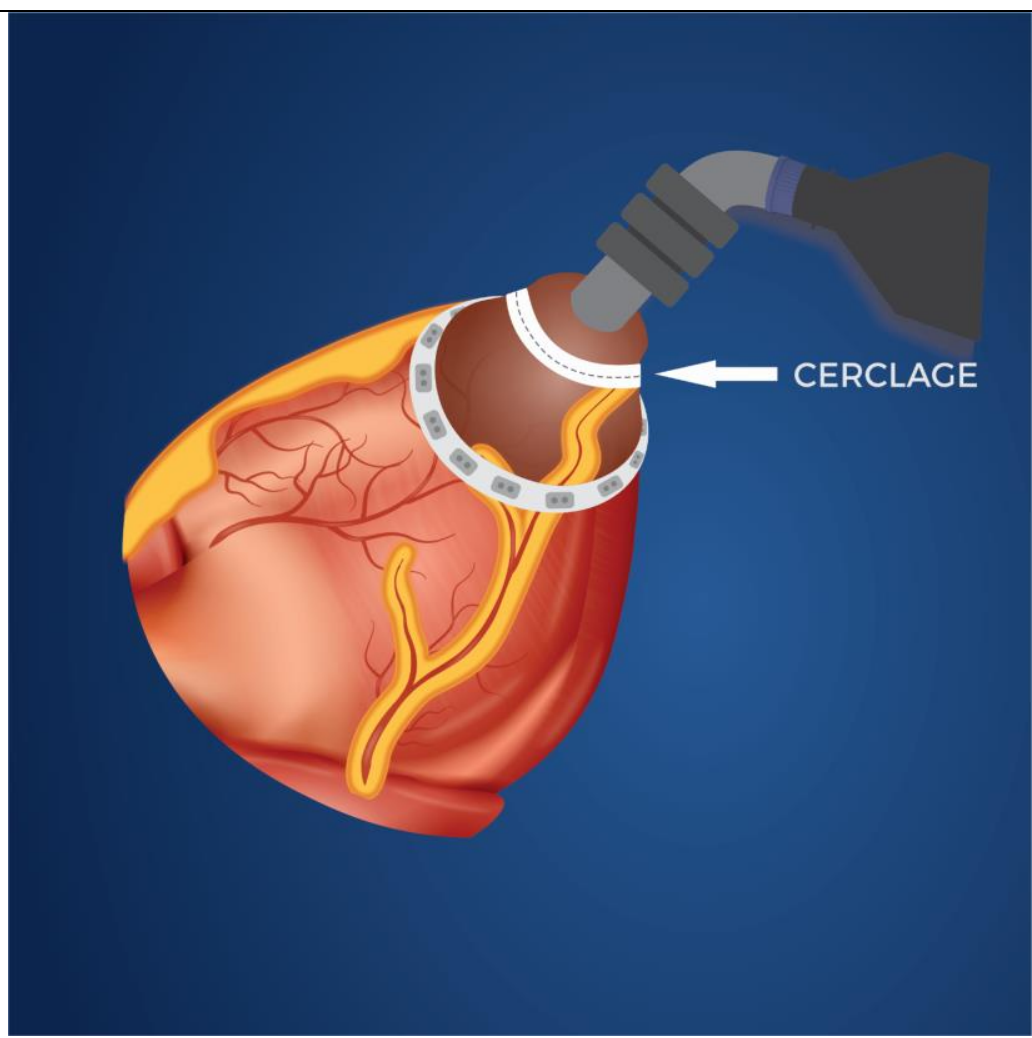

Figure 4. Cerclage using purse string suture reinforce with felt strip around the epicardium

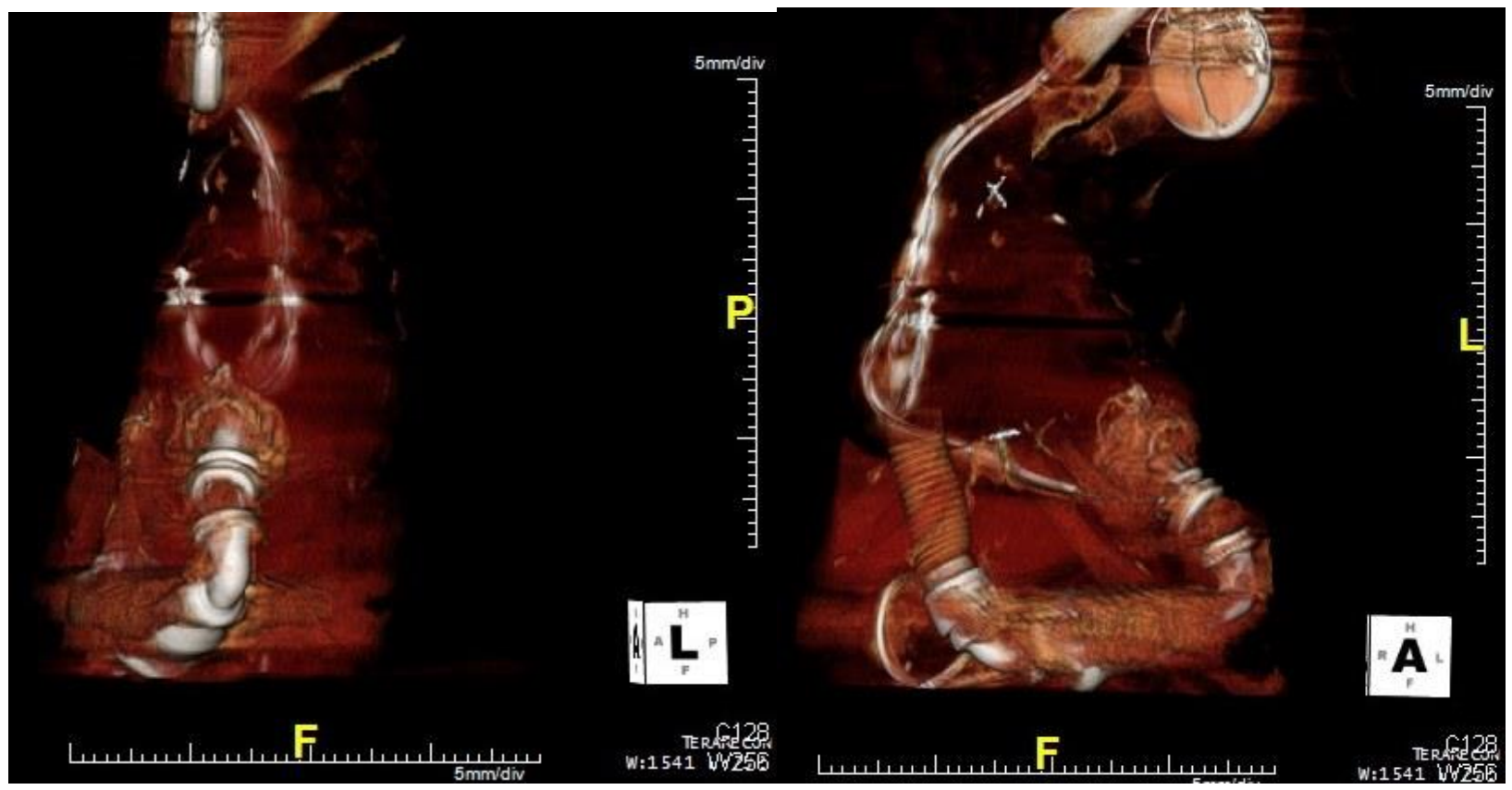

Figure 5. CT chest with $3 \mathrm{D}$ reconstruction showing inflow cannula through calcified apex. 


\section{Discussion}

A heavily calcified LV apex should not impede the implantation of an intracorporeal LVAD. Calcification is easy to determine preoperatively by imaging and should be recognized in advance to allow adequate surgical planning and a definitive approach.

Generally, for paracorporeal temporary assist devices, the inflow cannula could be placed in the left atrium, although ventricular cannulation generates better flow from a determined speed (7). However, the most common inflow site location for intracorporeal LVADs is at the LV position. In cases of extensive disease on the LV apex, the preferred choice for inflow cannulation remains the LV apex by either true apical implantation or by the diaphragmatic LV surface near the true LV apex. The most distal aspect of the base of the LV diaphragmatic surface is near the base of the heart, which consists of papillary muscles and the mitral apparatus. However, inserting the inflow cannula near those anatomical features could potentially occlude the inflow cannula (8). In our case, the diaphragmatic surface adjacent to the true apex was also heavily calcified.

The surgical technique described above represents a different and simplified surgical option in rare cases of a heavily calcified LV apex. Our technique differs from other proposals as we performed a non-excisional technique by preserving the LV apex and its calcification.

In our view, excisional techniques are acceptable, but we recognize that they could potentially lead to intraoperative issues. On one hand, the radical excision of the calcified LV apex could leave a small LV cavity; this could impact the inflow cannula positioning and LVAD function. On the other hand, the need for reconstructive $L V$ techniques, like patch elongation, is time consuming, which translates into longer CPB support time and potentially more platelet dysfunction, low fibrinogen levels and coagulopathy (9). In addition, there could be an added need for blood product transfusions that could potentially affect the performance of the right ventricle, which in some cases has deleterious impacts on the overall survival. The more extensive excisional techniques (removing the mitral apparatus and creating a single cavity) could potentially affect the LVAD hemodynamics.

After reviewing the literature and based on our knowledge, current techniques apply excisional technique when the entire calcified ventricular apex is excised. Our proposal is to maintain the calcific area by coring just the inflow site, thereby preserving the spherical shape and large geometry of the dysfunctional LV for better positioning of the inlet cannula parallel to the septum and directed to the mitral valve. The addition of a second suture line using a felt strip with partial thickness bites (epicardium) around the inlet in a purse string fashion enhances hemostasis around the inflow insertion site and stays away from the calcium, which sits in a deeper layer. The procedure is not time consuming and reinforces the tissue around the inlet site. 
In conclusion, treating patients with advanced heart failure and LV apical calcification requiring intracorporeal LVAD support is an infrequent occurrence. A simple and hemostatic, non-excisional technique for inflow placement is presented could be applied when advanced heart failure surgeons face this challenging and unique problem.

\section{Acknowledgement}

The authors would like to thank Dr. Michelle Sauer Gehring and Marina Machado for their editorial and graphic assistance.

\section{References}

1. Boyle AJ, Ascheim DD, Russo MJ, Kormos RL, John R, Naka Y, et al. Clinical outcomes for continuous-flow left ventricular assist device patients stratified by pre-operative INTERMACS classification. J Heart Lung Transplant. $2011 ; 30(4): 402-7$.

2. Adamson RM, Bower BL, Sundareswaran KS, Farrar DJ, Dembitsky WP. Radiologic assessment of HeartMate II position: Minimal pump migration after long-term support. J Heart Lung Transplant. 2015;34(12):1617-23.

3. Maltais S, Kilic A, Nathan S, Keebler M, Emani S, Ransom J, et al. PREVENtion of HeartMate II Pump Thrombosis Through Clinical Management: The PREVENT multi-center study. J Heart Lung Transplant. 2017;36(1):1-12.

4. Atluri P, Dymond DJ, Woo YJ. Continuous-flow left ventricular assist device implantation in the presence of a hostile ventricular apex. J Thorac Cardiovasc Surg. 2013;146(4):981-2.

5. Palmen M, Verwey HF, Haeck ML, Holman ER, Schalij MJ, Klautz RJ. Implantation of a left ventricular assist device in patients with a complex apical anatomy. Ann Thorac Surg. 2012;94(6):2122-5.

6. Frazier $\mathrm{OH}$, Gregoric ID, Messner GN. Total circulatory support with an LVAD in an adolescent with a previous Fontan procedure. Tex Heart Inst J. $2005 ; 32(3): 402-4$.

7. Timms D, Gregory S, Hsu PL, Thomson B, Pearcy M, McNeil K, et al. Atrial versus ventricular cannulation for a rotary ventricular assist device. Artif Organs. 2010;34(9):714-20.

8. Gregoric ID, Cohn WE, Frazier OH. Diaphragmatic implantation of the HeartWare ventricular assist device. J Heart Lung Transplant. 2011;30(4):467-70. 
9. Erdoes G, Gerster G, Colucci G, Kaiser H, Alberio L, Eberle B. Prediction of Post-Weaning Fibrinogen Status during Cardiopulmonary Bypass: An Observational Study in 110 Patients. PLoS One. 2015;10(5):e0126692. 